\title{
Coordination of care in hospitals: A rapid review of the literature
}

\author{
Dominic Madell, Luis Villa, Brooke Hayward, Lyndsay Le Comte \\ Ko Awatea, Counties Manukau District Health Board, New Zealand
}

Received: July 14, 2015

Accepted: September 5, 2015

Online Published: September 9, 2015

DOI: $10.5430 /$ jha.v4n6p77

URL: http://dx.doi.org/10.5430/jha.v4n6p77

\begin{abstract}
Project SWIFT (System Wide Integration for Transformation) is a programme of work supported by developments in technology, that aims to improve the health of people in Counties Manukau through initiatives focused on community-based care and improving hospital systems. A "rapid review" of literature focussing on coordination of care in hospitals was carried out to support this project. Rapid review is a literature review methodology that is "streamlined" by limiting: the number of databases searched, the types of study design included, the languages that articles are written in, the dates when articles were written, and the level of inclusion of "grey" literature. In total, 30 articles were considered in detail for inclusion in this rapid review, with many other articles considered briefly from title or abstract alone. Of the 30 articles, $12(40 \%)$ were ultimately deemed relevant, and included. In total, 112 unique articles contributed to the literature review, if all of the articles considered by three systematic reviews that contributed to this rapid review are included. The review found reasonable evidence that implementation of patient care delivery models focussing on coordination of care and efficiency can contribute to reductions in length of stay for hospital patients. In addition, reasonable evidence was found indicating that that the use of preoperative briefings and surgical safety checklists by operating teams can improve patient safety outcomes. However, it was not possible to draw firm conclusions from many of the other articles that were reviewed: these tended not to describe measurable improvements to patient outcomes or efficiency, and instead focussed on results that were process rather than outcome oriented, subjective, reported improvements that were not compared against any other measure, or were non-significant.
\end{abstract}

Key Words: Workflow, Coordination, Orchestration, Efficiency, Improvement, Hospital

\section{INTRODUCTION}

Project SWIFT (System Wide Integration for Transformation) is a programme of work supported by developments in technology, that aims to improve the health of people in Counties Manukau through initiatives focused on community-based care and improving hospital systems. ${ }^{[1]}$ It is being carried out by the Counties Manukau District Health Board (DHB) in New Zealand over a four-year period. Counties Manukau DHB has a population that is younger than the national average. It has a slightly higher proportion of Māori (16.3\% vs. 15.4\%), a much higher proportion of Pacific people (23.2\% vs. 6.7\%), and more people in the most deprived section of the population (Ministry of Health, 2015). A literature review was carried out to contribute to an aim of Project SWIFT that was to coordinate care and workflow management in hospitals, and standardise hospital processes to result in patients being less likely to suffer from adverse medical events. These objectives required a review of New Zealand and international research literature to be carried out.

\footnotetext{
*Correspondence: Dominic Madell; Email: dominic.madell@middlemore.co.nz; Address: Ko Awatea Research and Evaluation Office, Level 1 Esmé Green Building, Middlemore Hospital, 100 Hospital Road, Otahuhu, Private Bag 93311, Auckland 1640, New Zealand. 


\section{Methods}

A rapid review methodology was used for this literature review. This is a streamlined literature review process that may be shortened by, for example, limiting the number of databases searched, the types of study design included, the languages that articles are written in, the dates when articles were written, and the level of inclusion of "grey" literature. Also, the number of reviewers involved in decisions about inclusion and exclusion of articles, data extraction and quality assessment may be fewer than normal. ${ }^{[2]}$ Because of this streamlined procedure, the limitations of rapid review can include selection bias, publication bias and language of publication bias. However, the methodology was viewed as appropriate for this review due to the limited time and resources available, and it provided a good balance between costs and benefits.

The methodology consisted of identifying and selecting publications that were relevant to the area of focus, critically appraising publications, analysing the data reported, and describing results. First, inclusion and exclusion criteria were defined to guide which articles to include in the literature review. Articles included were those that reported research relating to any of the following within a hospital setting: integration of tasks and workflow, optimisation of clinical or allied health resources, prevention of readmission through integrated care or standardisation of processes, increased clinical or allied health efficacy, reduction in clinical or other staff, standardisation of hospital processes, and reduction of patient adverse medical events through optimisation of processes. Any research with a publication date from 2005 to 2015 that was written in English, and with any study design, was included. Excluded articles included non-research articles, those that reported research relating to General Practice (GP) or community services, those that reported benefits achieved through improvements to technical or clinical skills, articles which described process modelling or simulation techniques but did not apply these techniques to a real-life setting, and non peer-reviewed articles that were carried out outside of New Zealand.

Standard search engines (Medline Complete, Ebsco Health Business Elite, Cochrane, Health Foundation Research Scan, Heath Improvement and Innovation Resource Centre portal, Google Scholar, Pub Med, Science Direct, Web of Knowledge) were used to search for articles using combinations of the following terms: "integrated workflow", "workflow orchestration", "workflow improvement", "process improvement", "workflow", "efficiency", "coordination of hospital care", "orchestration of hospital care", and "efficient hospital care". Titles or abstracts of papers found were checked and non-relevant literature was removed, with five percent of non-relevant articles being "audited" by a second reviewer to check for agreement. As articles were read, further references were also sought from any relevant papers found using a snowballing technique.

The papers included were then critically reviewed by providing a written summary of methods, findings and conclusions from each of the articles, and summarising the limitations of the research in terms of their applicability to the hospital context. The quality of evidence in each article was scored on a number of variables relevant to the project: study design, participant characteristics, health system in which the research was carried out, population studied, and outcome measured. All variables were scored from zero to three. Study design, health system, and outcome measured were then weighted by a factor of two, as these were considered the most important variables for the review. The scoring system resulted in a total score out of 24 for each article, where 0-7 was defined as weak evidence, $8-15$ as moderate evidence, and 16-24 as strong evidence.

For study design, three points were awarded to systematic reviews that included at least one randomised controlled trial (RCT), or research or evaluation studies with experimental designs that included randomisation; two points were awarded to systematic reviews that did not include RCTs, prospective cohort studies, or evaluations with an experimental design but without randomisation; one point was awarded to retrospective cohort studies, case-control studies or evaluations with before/after designs, or that described baseline data; and no points were awarded to cross sectional surveys, evaluations without comparison groups and qualitative research.

For the participant characteristics category, studies which featured participants from a range of clinical disciplines and measured patient perspectives were awarded three points, studies which featured participants from a range of clinical disciplines only were awarded two points, studies which featured participants relating to a single clinical discipline were awarded one point, and studies which did not define participants were awarded zero points.

For the health system category, studies which reported research that was carried out in settings most similar to that found in New Zealand were awarded higher scores. Therefore, three points were awarded to studies carried out in public hospitals, two points were awarded to studies that were carried out in hospitals that were publicly funded but privately run, and zero points were awarded to studies carried out in private hospitals, or where there was no description of the setting (there was no one point score available for this category). 
For the "population" score, three points were awarded to studies that demonstrated an impact across an entire hospital, two points were awarded to studies that demonstrated an impact across a number of healthcare domains within a hospital, one point was awarded to studies that demonstrated an impact in a single healthcare domain, and no points were awarded to studies that poorly defined the type of healthcare domain in which the intervention had occurred.

Finally, in terms of outcome measured, studies that included measures relating to efficiency and quality of care received three points, studies that included measures relating to efficiency or quality of care received two points, studies that included only measures of the extent of usage of a system, or measured subjective user perceptions or satisfaction with a system received one point; and studies which did not include any measures received zero points.

Scores were interpreted cautiously, and were mainly used as a way of presenting the strength of the evidence in an easyto-read format. A further "audit" of the scores from $10 \%$ of the included articles was carried out by a second reviewer, to check for agreement.

\section{Results}

In total, 30 articles were read in full to assess their relevance for inclusion in this review, with many other articles being considered briefly from title or abstract alone. Of these 30 articles, 12 (40\%) were judged relevant and included. Of the 12 articles, three were systematic literature reviews, one was an RCT, one was a study with a control group (but where randomisation had not been used), one was a study that used pre/post data, five were studies where quantitative or qualitative data had been collected but that was not compared with anything, and one was a study that reported a planned RCT which had not yet been carried out.

Studies were carried out in a diverse range of international hospitals in New Zealand, England, Canada, USA and The Netherlands. In total, 112 unique studies contributed to the literature review, if all of the studies considered by the three systematic reviews are included. The average score out of 24 for these articles was 10.75, and the range of scores is illustrated in the box plot in Figure 1. The 12 included articles will now be discussed.

In general, the literature review found evidence that implementation of patient care delivery models focussing on coordination of care and efficiency can reduce length of stay for patients. Hajewski and Shirey ${ }^{[3]}$ evaluated the implementation of a patient care delivery model in an acute care hospital with 60 beds, using a pre-post study design. The goal of the model was to increase coordination of care between staff nurses and nurse case managers, and streamline care delivery through review of roles and tasks, and use of automation and electronic medical records. A control unit acted as a comparison site: this was a 44 bed medical surgical orthopaedic trauma unit that was similar to the intervention unit in terms of size, patient population and makeup of team members. The study found that length of stay decreased significantly for those in the intervention unit, but not for those in the control unit (mean length of stay [days] over a quarter for patient-care delivery model group: pre $=6.02$, post $=5.02$, $p=.049$; for control group: pre $=5.19$, post $=5.11, p=.45$ )

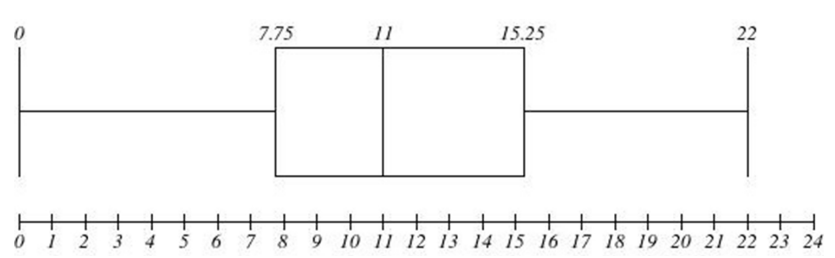

Figure 1. Box-plot showing scores awarded to articles included in rapid review

The literature review also highlighted evidence suggesting that the use of preoperative briefings and surgical safety checklists by operating teams can improve patient safety outcomes. Weller and Boyd ${ }^{[4]}$ undertook a systematic review of 46 studies of interventions to improve teamwork and communication in the operating room and highlighted two key studies supported by strong evidence. The first was by the Veterans' Health Administration who provided a programme of operative team training that included instruction in briefing, which was evaluated retrospectively against a control group. A significant decline in the risk-adjusted surgical mortality rate in the trained group was found as compared with the non-trained group $(\mathrm{RR}=1.49 ; 95 \% \mathrm{CI}, 1.10-2.07$; $p=.01)$. In addition, for every quarter of the training program delivered, a reduction of 0.5 deaths per 1,000 procedures occurred (95\% CI, 0.2-1.0; $p=.001) .{ }^{[5]}$ The second key study was a controlled trial of the Surgical Patient Safety System (SURPASS) checklist at six intervention hospitals in The Netherlands, compared with five control hospitals. ${ }^{[6]}$ In the intervention hospitals, the proportion of patients with one or more complications decreased from $15.4 \%$ to $10.6 \%$ $(p<.001)$, while there was no change in the control hospitals.

An article found in the review that is worthy of mention because of the strong study design it described, but where results are not yet available, was written by Meltzer and Ruhnke, ${ }^{[7]}$ who described a Comprehensive Care Physician (CCP) mode 1. In this model, physicians provide both inpatient and outpatient care to their patients to reduce the discontinuities in care that can arise with the use of separate "hospitalists" and primary care physicians. Meltzer and 
Ruhnke report that an RCT will be carried out to assess this model's effects on costs and outcomes, and the results of this will be available in 2016. ${ }^{[7]}$ Given the high quality of design that is described, this study would be worth reviewing when the results are released.

The other research found in this review that described possible methods for improving care coordination in hospitals included literature that focussed on: the use of communications technology to improve clinical communications, ${ }^{[8]}$ the effectiveness of quality improvement and other interventions taking place in acute care settings (a systematic review), ${ }^{[9]}$ studies that considered the effectiveness of information display systems in hospitals, ${ }^{[10-12]}$ studies that considered the use of electronic task management tools, ${ }^{[13,14]}$ a study that considered the effectiveness of nurse clipboard design, ${ }^{[15]}$ and one that looked at the effectiveness of using a secure messaging application in a hospital. ${ }^{[16]}$ However, as a group, this research did not describe measurable improvements to patient outcomes or efficiency, instead focussing on results that: were process rather than outcome oriented (such as the extent of usage of tools, or impacts on team behaviours); were subjective (such as measures of user satisfaction and self-efficacy, attitudes towards teamwork, and perceptions of care); reported improvements that were not compared against any other measure; or were statistically non-significant.

\section{Discussion}

The priority of this review was to find articles that reported either a high quality of evidence or summarised a large quantity of evidence. However, it is acknowledged that not all articles of relevance may have been identified due to the rapid review methodology employed. Nevertheless, research suggested that if operating teams changed their practice from using no or limited briefing to using a high quality preoperative briefing, and to using surgical safety checklists, this could improve patient and safety outcomes. This finding is of particular interest because it illustrates that the implementation of certain measures to coordinate care in hospitals can be relatively simple, with modest resource implications, but still have potentially far-reaching impacts on patient health.

The review also presented reasonable evidence that implementation of patient care delivery models that increase the coordination of care, eliminate rework and redundancy, and streamline care delivery processes might be effective in reducing length of stay for patients. However, the review could not identify which specific aspects of such patient care delivery models would be of the most benefit to hospitals, and which aspects would be of lesser importance. Given the potential financial and other resource implications of implementing such interventions, this would seem to be an important issue for future research to address. Unfortunately, literature tended to consider outcomes from sets of interventions delivered as a group, and the individual merits of each strand were not teased out. In future, patient care delivery models that are implemented in hospitals could be carefully evaluated with this issue in mind. Findings from such robust evaluations would not only be valuable for the hospitals in which the model was implemented, but also for other hospitals who are interested in taking similar courses of action.

\section{Conclusion}

The findings from this literature review will be considered in context alongside many other initiatives that contribute to Project SWIFT, such as quality improvement investigations, evaluations of systems and services, engagement with patients, and improvements to IT systems. This literature review will contribute to the project's "detailed design" phase, which involves developing the overall SWIFT program of work: the phase ends with the presentation of a number of business cases for change to the Counties Manukau DHB.

This review has highlighted the importance of patient care delivery models and the use of surgical safety checklists and pre-operative briefings in hospitals, and suggests that future research relating to care coordination in hospitals will be of most use if it demonstrates strong evidence that relates to patient health outcomes, or efficiency in the use of resources. While other kinds of result are also of interest, these should ultimately be reported in support of these strong indicators of benefit.

\section{ACKNOWLEDGEMENTS}

We thank the Ko Awatea library staff who carried out initial literature searches for Project SWIFT, and Wing Cheuk Chan and Doone Winnard of the Counties Manukau DHB Population Health Team who provided peer review of the articles.

\section{REFERENCES}

[1] Project SWIFT [Internet]. New Zealand, Counties Manukau Health [cited 2015 Jul 7]. Available from: http: //www. countiesmanukau. health.nz/assets/About-CMH/P
rojects/SWIFT/CMDHB-About-Swift-2015.03-V5-LR.pdf

[2] Methods: Synthesis 1. Rapid reviews: Methods and implications. National Collaborating Centre for Methods and Tools [Internet] Canada, Ontario, Hamilton [cited 2015 Jul 7]. Available from: 
http://www.nccmt.ca/pubs/Methods_Synthesis1.pdf

[3] Hajewski CJ, Shirey MR. Care Coordination. A Model for the Acute Care Hospital Setting. Journal of Nursing Administration. 2014; 44(11): 577-585. PMid: 25340922. http://dx.doi.org/10.10 $97 /$ NNA . 0000000000000129

[4] Weller J, Boyd M. Making a difference through improving teamwork in the operating room: A systematic review of the evidence on what works. Current Anaesthesiology Reports. 2014; 4: 77-83. http://dx.doi.org/10.1007/s40140-014-0050-0

[5] Neily J, Mills PD, Young-Xu Y, et al. Association between implementation of a medical team training program and surgical mortality. Journal of the American Medical Association. 2010; 304: 1693-1700. PMid: 20959579. http://dx.doi.org/10.1001/jama.2010.1 506

[6] de Vries EN, Prins HA, Crolla RMPH, et al. Effect of a comprehensive surgical safety system on patient outcomes. New England Journal of Medicine. 2010; 363: 1928-1937. PMid: 21067384. http://dx.doi.org/10.1056/NEJMsa0911535

[7] Meltzer DO, Ruhnke GW. Redesigning care for patients at increased hospitalization risk: the comprehensive care physician model. 2014; 33(5): 770-777.

[8] Wu RC, Tran K, Lo V, et al. Effects of clinical communication interventions in hospitals: a systematic review of information and communication technology adoptions for improved communication between clinicians. International journal of medical informatics. 2012; 81(11): 723-732. PMid: 22727613. http://dx.doi.org/10.1016/j.i jmedinf . 2012.05.014

[9] Buljac-Samardzic M, Dekker-van Doorn CM, van Wijngaarden JDH, et al. Interventions to improve team effectiveness: A systematic review. Health Policy. 2010; 94: 183-195. PMid: 19857910. http://dx.doi.org/10.1016/j.healthpol.2009.09.015
[10] Wong H, Caesar M, Bandali S, et al. Electronic inpatient whiteboards: Improving multidisciplinary communication and coordination of care. International Journal of Medical Informatics. 2009; 78: 239-247. PMid: 18786851. http://dx.doi.org/10.1016/j.ijmedinf . 2008.07 .012

[11] Morton T. Emergency Department at a Glance: Nelson Marlborough District Health Board. directly emailed [not yet publicly accessible]. 2014.

[12] Wears RL, Perry SJ, Wilson S, et al. Emergency department status boards: user-evolved artefacts for inter- and intra-group coordination. Cognition, Technology and Work. 2007; 9: 163-170. http://dx.doi.org/10.1007/s10111-006-0055-7

[13] Kolowitz BJ, Lauro GR, Barkey C, et al. Workflow continuity - moving beyond business continuity in a multisite 24-7 healthcare organization. Journal of digital imaging. 2012; 25(6): 744-750. PMid: 22766799. http://dx.doi.org/10.1007/s10278-012 $-9504-4$

[14] Foo E, McDonald R, Savage E, et al. Mobile task management tool that improves workflow of an acute general surgical service. ANZ Journal of surgery. 2015 [EPub]. PMid: 25645082. http: //dx.doi.org/10.1111/ans. 12988

[15] Gurses AP, Xiao Y, Hu P. User-designed information tools to support communication and care coordination in a trauma hospital. Jounral of Biomedical Informatics. 2009; 42: 667-677. PMid: 19298868. http://dx.doi.org/10.1016/j.jbi.2009.03.007

[16] Przybylo JA, Wang A, Loftus P, et al. Smarter hospital communication: secure smartphone text messaging improves provider satisfaction and perception of efficacy, workflow. Journal of Hospital Medicine. 2014; 9(9): 573-578. PMid: 25110991. http: //dx.doi.org/10.1002/jhm.2228 